\title{
R. Brinkmann
}

Deutsches Elektronen-Synchrotron DESY

Notkestr. 85, 2000 Hamburg 52, W.-Germany

\section{Abstract}

The optics for the $350 \mathrm{~m}$ long proton beam transport system from PETRA to HERA is presented. The special geometry of the beam line, involving both horizontal and vertical deflections, leads to a coupling of horizontal and vertical emittances. A simple compensation scheme using skew quadrupoles is proposed.

The requirements for the magnets with respect to apertures, tolerances and flexibility (for matching various optics of PETRA and HERA) are discussed. An estimate for the emittance growth due to focussing errors in the beam line is given.

\section{General Layout and Geometry}

The injection and acceleration system for the e-p storage ring HERA (fig. 1) will include several of the existing DESY machines. In particular both electrons (at $14 \mathrm{GeV}$ ) and protons (at $40 \mathrm{GeV}$ ) will be injected

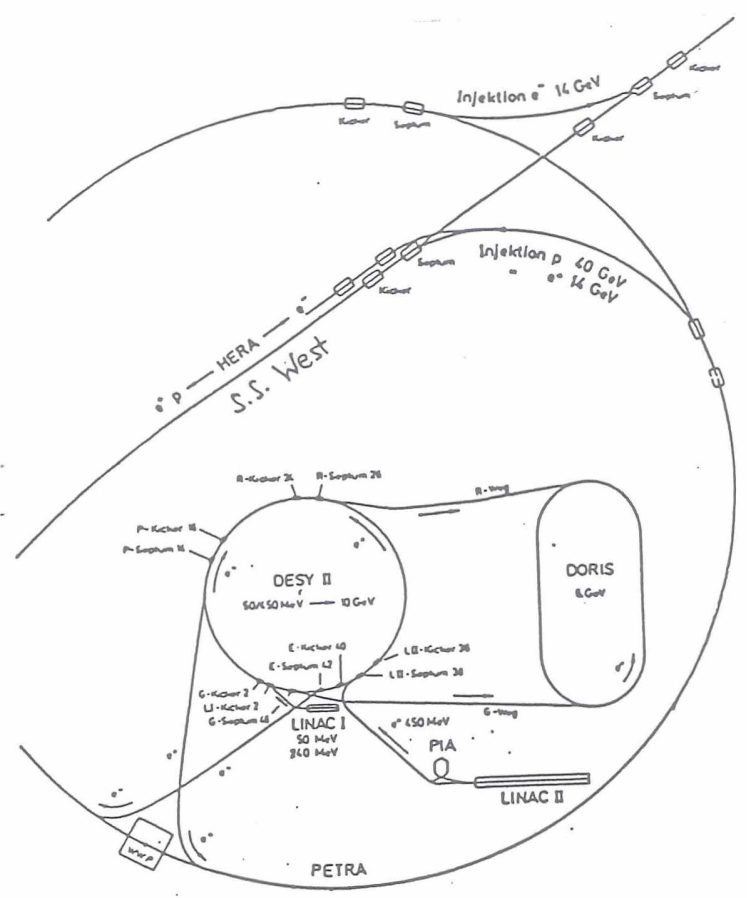

Fig. 1: The injector scheme for HERA

from the $e^{+}-e^{-}$storage ring PETRA / $1 /$. Therefore two new beam transfer lines from PETRA to HERA have to be built. Only the proton transfer line will be considered here. This line will also be used for positron injection at $14 \mathrm{GeV}$ for the $\mathrm{e}^{+} \mathrm{e}^{-}$operation mode of HERA.

The layout of the transfer line is mainly determined by geometrical boundary conditions. From the PETRA straight section North where the protons are ejected (fig.2) to the HERA straight section West where injection takes place (fig.3), the beam line must bend by a total of $110^{\circ}$ on the horizontal plane and has to descend by some $14 \mathrm{~m}$ to the level of HERA. Owing to further geometrical constraints, this bend angle must be achieved by means of two arcs of rather small mean radius $(\bar{R}=89 \mathrm{~m})$. At $e_{p}=40 \mathrm{GeV}$ this requires strong dipoles with $B=1.95$ T. Both arcs are interrupted by straight sections where the beam pipe crosses the tunnel walls, see fig's. 2 and 3, and they are linked by a $140 \mathrm{~m}$ long straight section. The necessary vertical deflections are located just outside the PETRA tunnel and near to the end of the line inside the HERA tunnel, respectively. The latter deflection would be turned off for positron injection. Positrons would then be injected into the HERA-e ring some $140 \mathrm{~m}$ downstream the centre of the straight section West at which point the e-beam is located $0.81 \mathrm{~m}$ below the p-beam $/ 2 /$.

As a consequence of the relatively strong vertical deflections and the sharp horizontal bends near the beginning and the end of the transfer line the two arcs lie in two different non-parallel planes so that across the whole line the beam coordinate system rotates about the longitudinal axis /3/ by about $6^{\circ}$. The consequences of this mismatch for the optics are discussed in the following section.

\section{Optics}

In the arcs of the transfer line the optics consists of a periodic FODO structure with a 13.5 m long cell (fig. 4). The periodic B-functions and horizontal dispersion reach $23 \mathrm{~m}$ and $2.2 \mathrm{~m}$, respectively, for a phase advance of $72^{\circ}$ per cell. At the ends of the arcs a match between the FODO structure and the optics of PETRA /4/ and HERA /2/

HORIZONTAL

DISPLRCEMENT [M]

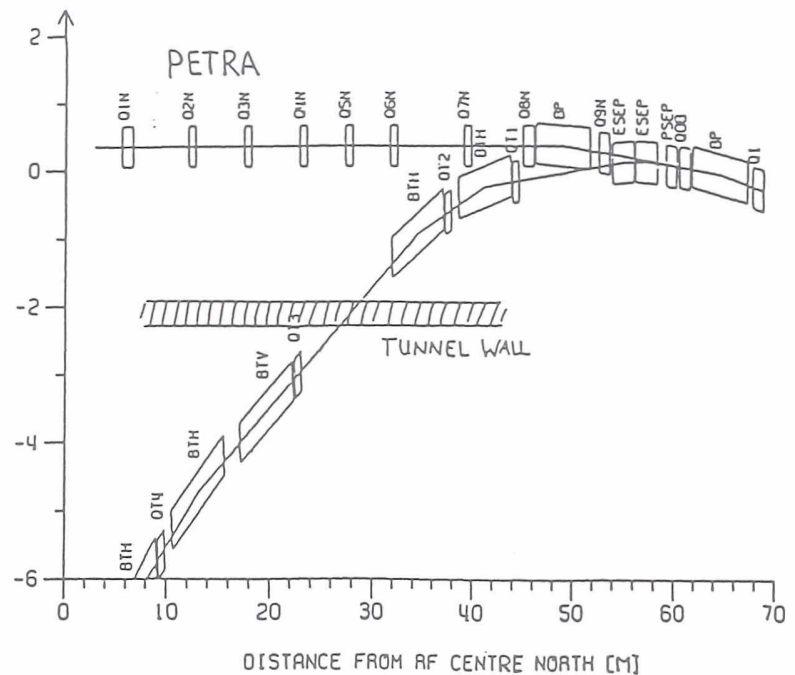

Fig. 2: Geometry in the horizontal plane of the transfer line near PETRA 

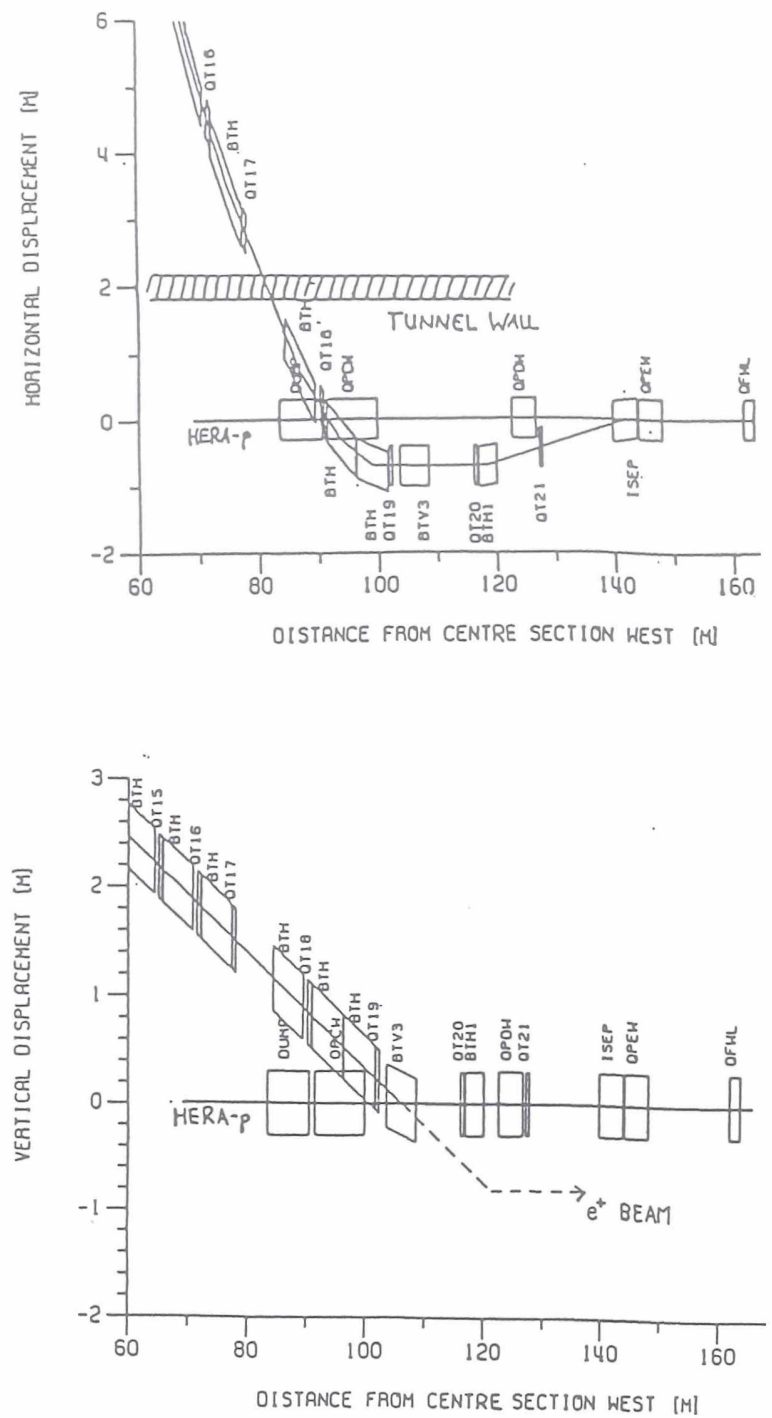

Fig. 3: Geometry of the transfer line near to HERA in the horizontal and vertical plane

has to be performed and for this a total of 15 individually variable quadrupoles is required. In the matching sections the B-values and dispersion reach $100 \mathrm{~m}$ and $4 \mathrm{~m}$, respectively.

In the long straight section of the beamline a simple symmetric doublet focussing system is used. The maximum $\beta$-function is about $250 \mathrm{~m}$ (see fig. 4). As has been previously mentioned, the planes in which the two arcs lie meet with a relative twist angle of $6^{\circ}$ in the straight section. In order to avoid a coupling of horizontal and vertical emittances and a mismatch to the HERA optics, this angle is compensated by slightly rotating the quadrupoles of the doublets about the s-axis so that the beam cross-section is also rotated by $6^{\circ}$ and emerges at HERA untwisted. Cecause of the symmetry of the optics, only two independent rotation angles are required to decouple the $4 \times 4$ transfer matrix for the section between the end of the first and the beginning of the second arc (this scheme is very similar to the method for compensation of an experimental solenoid using pairs of skew quadrupoles /5/).

The apertures required in the transfer line are determined by the expected beam emittances of $\varepsilon=\varepsilon=0.8 \times 10^{-6} \mathrm{~m}$ for the whole beam and a total

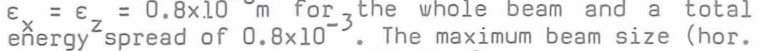
$x$ vert.) will then be $22 \times 18 \mathrm{~mm}^{2}$ in the arcs and $32 \times 36 \mathrm{~mm}^{2}$ on the straight section doublets. The magnets, which have now been designed $/ 6 /$, allow for an aperture of $45 \times 35 \mathrm{~mm}^{2}$ so that a safety factor sufficient for orbit deviations and (eventually) larger emittance is included. For the doublets in the straight section special quadrupole types will be used.

\section{Tolerances}

\section{Orbit Errors}

The main sources of orbit deviation in the transfer line are errors in the integrated field strength B.l of the dipoles and transverse position errors $\Delta \xi(\xi=x, z)$ of the quadrupoles. Reasonable values for these errors are $\langle B \cdot 1 / B \cdot 1\rangle_{\text {rms }}=5 \cdot 10^{-4}$ and $\langle\Delta \xi\rangle_{\text {rms }}=0.3 \mathrm{~mm}$. As a result of the sum of the perturbative kicks (assumed uncorrelated) that the beam receives from these errors, the rms orbit deviation at the position send of the injection septum will be $\langle\Delta x\rangle$ rms $=15 \mathrm{~mm}\left(B_{x}\left(s_{\text {end }}\right)=60 \mathrm{~m}\right)$ and $\langle\Delta z\rangle_{\text {rms }}=8 \mathrm{~mm}\left(\beta_{z}\left(s_{\text {end }}\right)=30 \mathrm{~m}\right)$. In order to correct the orbit errors, beam monitors and correction coils will be placed near each quadrupole in the transfer line.

\section{Focussing Errors}

Gradient errors in the quadrupoles will cause a perturbation of the optics leading to a mismatch at the end of the transfer line and resulting in a dilution of transvers phase space after injection into HERA. We give here a brief summary of the calculation of the expected emittance growth using a Hamiltonian formalism in action angle variables. The mean grouth of emittance is calculated from the perturbed action variable $J(s)=J+\Delta J(s)$ for a single particle (at the beginning of the beamline, $J(0)=J$ is equal to the emittance $\varepsilon_{0}$ of the particle).

Let $H(s)=H_{(s)}+H_{1}(s)$ be the perturbed Hamiltonian for betatron motion where $H_{0}(s)$ describes the unperturbed optics and

$$
H_{l}(s)=\frac{1}{2} k(s) B_{0}(s) J(s) \cos \left(\phi_{0}(s)+\phi_{1}(s)+\varphi\right)
$$

is the perturbation due to focussing errors $\Delta k(s)$. In (1), $\beta$ (s) is the unperturbed $\beta$-function, $\phi(s)$ is the perturbation of the phase advance along the beamline and $\varphi$ is the initial phase of the particle. The perturbed variables satisfy the canonical equations (7)

$$
\frac{d J}{d s}=-\frac{\partial H_{1}}{\partial \phi_{1}} ; \quad \frac{d \phi_{1}}{d s}=\frac{\partial H_{1}}{\partial J}
$$

For small phase perturbations $\phi_{1}(s)$ equ. (2) can be linearized and solved straight forwardly. In thin lens approximation, the function $\Delta k(s)$ is given by

$$
k(s)=\sum_{n=1}^{N} \Delta\left(k_{i} l_{i}\right) \delta\left(s-s_{i}\right)
$$

Where $\mathrm{N}$ is the number of quadrupoles in the transfer line, $s$. are their longitudinal positions and $\Delta\left(k_{i} l_{i}\right)$ the errors of focusing strength which are assumed to be random uncorrelated numbers small compared to 


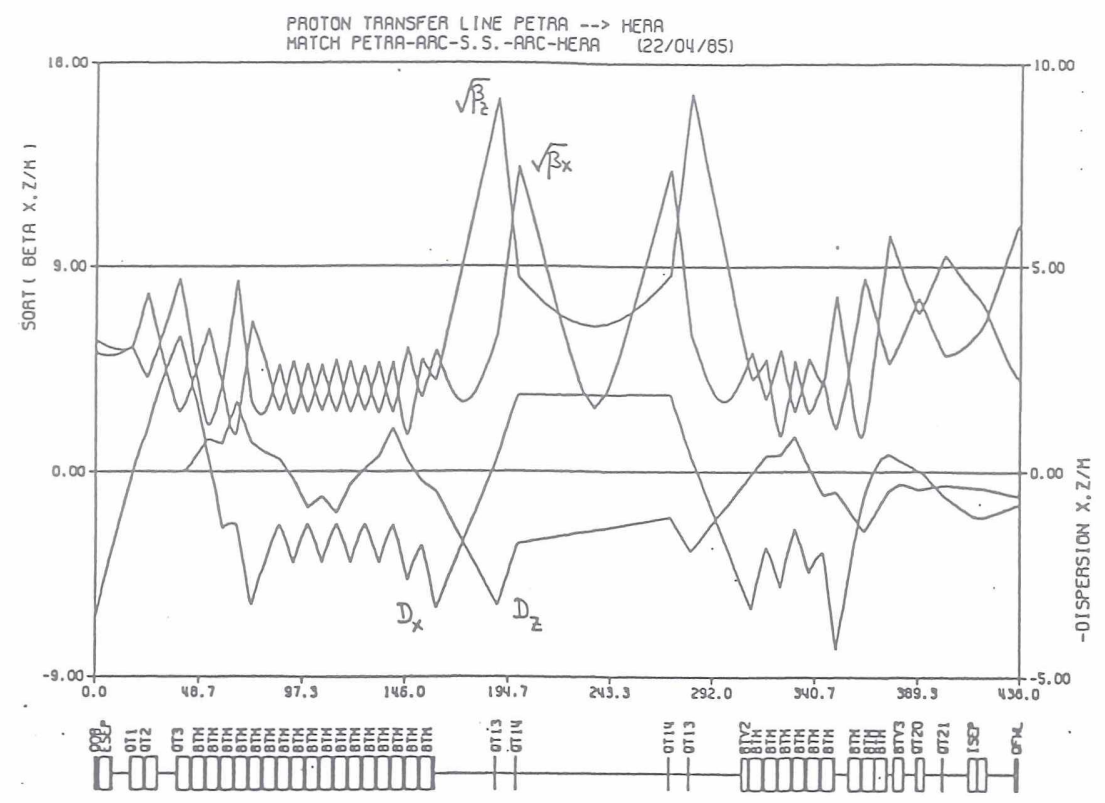

Fig. 4: Optics of the proton transfer line. The first and the last quadrupole are part of the PETRA and HERA lattice, respectively

the nominal strength of the quads. Putting (3) into the solution of (2), performing an average over the initial phase $\varphi$ and keeping only terms of lowest (quadratic) order of the gradient errors, we finally obtain for the mean value of $J$ at the end of the beamline:

$$
\left\langle J\left(s_{\text {end }}\right)\right\rangle=J_{0}\left(1+\frac{1}{2} \sum_{i=1}^{N}\left\langle\Delta\left(k_{i} l_{i}\right)^{2}>B_{0}^{2}\left(s_{i}\right)\right)\right.
$$

This corresponds to a mean value of the relative increase $\delta \varepsilon$ of emittance of

$$
\langle\delta \varepsilon\rangle=\frac{\left\langle J\left(s_{\text {end }}\right)\right\rangle}{J_{0}}-1=\frac{1}{2} \Gamma_{i=1}^{N}\left\langle\Delta\left(k_{i} I_{i}\right)^{2}\right\rangle \quad B_{0}^{2}\left(s_{i}\right)
$$

Since the B-function appears quadratically in eq. (5), the main contributions to $\langle\delta \varepsilon\rangle$ result from the straight section and from the matching sections at the beginning and the end of the transfer line. With relative accuracy of the quadrupole strengths of $10^{-3}$ the effective growth of emittance remains well below one percent in both planes. Since this accuracy can be easily attained, we do not expect gradient errors in the transfer line to make a major contribution to a luminosity reduction in HERA.

\section{Acknowledgements}

I am obliged to A. Febel, G. Meyer, J. RoBbach and F. Willeke for many helpful discussions. I also wish to thank D. Barber for careful reading of the manuscript.

\section{References}

1/ J. Maidment, "PETRA II, a proton injector for HERA", DESY HERA 83/12 (1983)

/2/ D. Barber, R. Brinkmann, R. Kose, J. Roßbach, K. Steffen and F. Willeke, "Layout and optics for HERA head on interaction region and straight sections", contribution to this conference

13/ K. Steffen, "Rotation of beam cross section in the HERA proton and positron injection channel", DESY HERA 84/01 (1984)

/4/ J. Roßbach, private communication

15/ K. Steffen, "Compensation of solenoid coupling with a single weak pair of quadrupoles", DESY HERA $82 / 11$ (1982)

/6/ A. Febel and F. Neubau, private communication

/7/ See e.g. H. Goldstein, "Classical Mechanics" (second edition), Addison-Wesley, Reading, Mass. (1980) 